\title{
Editorial
}

\section{Gauge/String Duality}

\author{
Wolfgang Mück, ${ }^{1}$ Carlos Núñez, ${ }^{2}$ Leopoldo A. Pando Zayas, ${ }^{3}$ \\ Alfonso V. Ramallo, ${ }^{4}$ Radoslav C. Rashkov, ${ }^{5,6}$ \\ and Kadayam S. Viswanathan ${ }^{7}$ \\ ${ }^{1}$ Dipartimento di Scienze Fisiche, Universitá degli Studi di Napoli Federico II, INFN, Sezione di Napoli, \\ Via Cintia, 80126 Napoli, Italy \\ ${ }^{2}$ Department of Physics, University of Wales Swansea, Singleton Park, Swansea SA2 8PP, UK \\ ${ }^{3}$ Michigan Center for Theoretical Physics, The University of Michigan, Ann Arbor, MI 48109-1120, USA \\ ${ }^{4}$ Departamento de Fisica de Particulas, Instituto Galego de Fisica de Altas Energias (IGFAE), \\ Universidade de Santiago de Compostela, 15782 Santiago de Compostela, Spain \\ ${ }^{5}$ Institute for Theoretical Physics, Vienna University of Technology, Wiedner Hauptstrasse 8-10, \\ 1040 Vienna, Austria \\ ${ }^{6}$ Department of Physics, Sofia University, 1504 Sofia, Bulgaria \\ ${ }^{7}$ Department of Physics, and IRMACS, Simon Fraser University, Burnaby, British Columbia, Canada
}

Correspondence should be addressed to Kadayam S. Viswanathan, kviswana@sfu.ca

Received 9 December 2010; Accepted 9 December 2010

Copyright (C) 2010 Wolfgang Mück et al. This is an open access article distributed under the Creative Commons Attribution License, which permits unrestricted use, distribution, and reproduction in any medium, provided the original work is properly cited.

In the past few years, we have witnessed rapid developments in the study of gauge/string dualities. The prototype of this duality is the AdS/CFT correspondence, originally conjectured as the duality between type IIB string theory in $\mathrm{AdS}_{5} \times S^{5}$ with $N$ units of R-R five-form flux and $\mathcal{N}=4 S U(N)$ super Yang Mills (SYM) theory in $d=4$ spacetime dimensions. There are many superb review articles on the subject of the AdS/CFT correspondence. More recently, the subject of holographic duality has been extended in diverse directions with surprising new results. One of these directions has been related to the applications of the holographic principles to realistic gauge theories which are not conformal such as QCD. It is widely agreed that an exact string dual to QCD is not known at present. Nevertheless, there are holographic models which share features of QCD such as confinement and deconfinement at high temperatures and chiral symmetry breaking, and restoration. However, these models do not exhibit the asymptotic freedom of QCD. They have, however, shed light on the properties of QCD in the strong coupling regime. Another area that has seen activity is the study of relativistic heavy ion collision (RHIC), where a quark-gluon plasma is formed. Holographic principles have also been studied in nonrelativistic systems that promise to be relevant for the study of phase transitions and other phenomena in inherently strongly coupled systems in condensed matter physics. 
This special issue brings together some of the latest developments in this field of research. The contributions cover both technical aspects and applications of the gauge/string dualities. Many of them contain new and original results which we acknowledge with thanks.

The paper by L. Alday titled "Scattering amplitudes, AdS/CFT correspondence, Minimal surfaces, and Integrability" discusses the computation of gluon scattering amplitudes in planar maximally supersymmetric Yang-Mills theory in four space-time dimensions at strong coupling by means of the AdS/CFT duality. This problem reduces to the computation of minimal surfaces in AdS. Integrability allows one to give a solution to the problem of computing scattering amplitudes in terms of a set of integral equations. The method is applied to 4-gluon scattering.

The next paper, "Generalized Gribov-Lipatov reciprocity and AdS/CFT" by M. Beccaria et al., reviews the Gribov-Lipatov reciprocity in the supersymmetric $N=4$ SYM theory by means of its dual gravity theory. Since the AdS/CFT correspondence links $N=4$ SYM and superstring dynamics in $\mathrm{AdS}_{5} \times S^{5}$, reciprocity is expected to show up in the quantumcorrected energies of certain classical string configurations dual to gauge theory operators. The authors review recent results confirming this picture and revisiting the old idea of Gribov-Lipatov reciprocity as a modern theoretical tool for the study of open problems in AdS/CFT.

The paper titled "The master space of supersymmetric gauge theories" by A. Zaffaroni and A. Hanany gives a review of the moduli space and the spectrum of chiral operators for gauge theories on branes at singularities. The focus is on theories with four real supercharges in $3+1$ and $2+1$ dimensions. These theories are holographically dual to $\mathrm{AdS}_{5} \times H^{5}$ and $\mathrm{AdS}_{4} \times H^{7}$ in type IIB or M theories, respectively, where $H^{5}$ and $H^{7}$ are Sasaki-Einstein spaces. Master space is roughly the moduli space for one single brane. The authors demonstrate that most of the information on the moduli space and the spectrum of quiver gauge theories are encoded in the concept of "master space" consisting of mesonic and baryonic degrees of freedom. Relevant information on the generated functions for chiral operators which can be computed using plethystics techniques and the language of complex geometry is summarized.

The paper by V. Marotta Puletti is titled "On string integrability: a Journey through the 2-dimensional hidden symmetries in the AdS/CFT dualities." The discovery of infinitely many conserved charges, that is, integrability in the planar AdS/CFT, has allowed us to make immense progress in understanding and confirming the AdS/CFT duality conjecture. In this paper, the fundamental concepts and properties of integrability of 2-d $\sigma$ models and in the AdS/CFT context are reviewed. The first part is focussed on $\mathrm{AdS}_{5} / \mathrm{CFT}_{4}$, the classical and quantum integrability of the type IIB superstring on $\mathrm{AdS}_{5} \times S^{5}$ are especially discussed in both pure spinor and Green-Schwarz formalism. The second part deals with $\mathrm{AdS}_{4} / \mathrm{CFT}_{3}$ with particular attention to type IIB superstrings on $\mathrm{AdS}_{4} \times \mathrm{CP}^{3}$ and its integrability.

The paper "The high spin expansion of twist sector dimensions: the planar $N=4$ super Yang Mills theory" by D. Fioravanti and M. Rossi is devoted to collecting some results on the high spin expansion of anomalous dimensions in planar $\mathcal{N}=4 \mathrm{SYM}$ or its $\mathrm{AdS}_{5} \times S^{5}$ string counterpart. The authors restrict the discussions to the twist operators, although some ideas and structures turn out to be general for other sectors or gauge theories.

In the sixth paper titled "Unquenched flavor in the gauge/gravity correspondence," A. Ramallo et al. review the studies of field theories with a large number of adjoint and fundamental fields in the Veneziano limit. Emphasis is placed on setups where the fundamentals are introduced by means of a smeared set of D-branes. First the general ideas are discussed and then particularities are considered in various models. The paper also contains new results. 
The seventh paper by V. Filev and R. Rashkov is titled "Magnetic catalysis of chiral symmetry breaking: a holographic perspective." The authors discuss recent investigations of the effect of magnetic catalysis of mass generation on holographic YM theories. The main part of the review is devoted to studying the effect of external magnetic field on holographic gauge theories dual to D3/D5 and D3/D7 brane intersections. Among the observed phenomena are the spontaneous breaking of a global internal symmetry, Zeeman splitting of the energy levels, and the existence of pseudo-Goldstone modes. An analytic derivation of the GellMann-Oaks-Renner relation for the D3/D7 setup is reviewed. In the case of D3/D5, the pseudo-Goldstone modes satisfy nonrelativistic dispersion relations. The studies reviewed confirm the universal nature of magnetic catalysis of mass generation.

The next paper is by M. Piai entitled "Walking technicolor, holography, and gauge/gravity dualities." It contains a discussion of dynamical symmetry breaking in electroweak theory as an appealing, strongly coupled alternative to the weakly coupled models based on elementary Higgs field developing a nonzero VEV. After summarizing the arguments based on low-energy phenomenology and supporting walking technicolor as a meaningful realization of dynamical symmetry breaking, a pedagogical introduction to walking technicolor and extensions of the standard model are presented. The strongly coupled nature of the underlying interactions and the peculiar quasiconformal behaviour of the theory requires one to use nonperturbative methods in order to address many fundamental questions within this framework. The developments in gauge/gravity dualities provide an ideal tool to study this scheme. Potential uses of these techniques are presented.

The next two papers deal with the subject of quark-gluon plasma and systems out of equilibrium. Felix Rust's paper is titled "In-medium effects in the holographic quark-gluon plasma." In this paper, the gauge/gravity duality is used to investigate various properties of strongly coupled gauge theories, which are interpreted as models for the quark-gluon plasma (QGP). In particular, variants of the D3/D7 setup as an implementation of the top-down approach of connecting string theory with phenomenologically relevant gauge theories are studied. Finite temperature and finite density effects on fundamental matter in the holographic quark-gluon plasma are studied. A setup in which one can describe the holographic plasma at finite temperature and either baryon or isospin density is used and the properties of the system are investigated from three different viewpoints is presented. Meson spectra, temperature, and density dependence of transport properties of fundamental matter in the QGP are calculated. The observations presented here are in qualitative agreement with phenomenological models and experimental observations.

V. E. Hubeny and M. Rangamani's paper entitled "A holographic view on physics out of equilibrium" is a review of recent developments in applying holographic methods to understand nonequilibrium physics in strongly coupled field theories. The emphasis is on elucidating the relation between the evolution of quantum field theories perturbed away from equilibrium and the dual picture of dynamics of classical fields in black hole backgrounds. The linear response regime, the hydrodynamic regime, and the non-linear regime of interacting quantum system are discussed. It is shown how the duality might be used to learn salient aspects of black hole physics in terms of field theory observables.

The paper by J. McGreevy "Holographic duality with a view toward many-body physics" is notes based on a series of lectures given at the KITP Workshop-Quantum Criticality and the AdS/CFT Correspondence in July, 2009. The goal of the lectures was to introduce condensed matter physicists to the AdS/CFT correspondence. Discussion of string theory and of supersymmetry is avoided to the extent possible. 
The final paper titled "Holographic renormalization of 2-point functions in non-AdS/Non$C F T^{\prime \prime}$ by M. Haack and W. Mück reviews recent progress on holographic renormalization in the context of the gauge-gravity correspondence when the bulk geometry is not asymptotically AdS. The prime example is the Klebanov-Strassler background, whose dual gauge theory has logarithmically running couplings at all energy scales. The presented formalism provides the counterterms necessary for obtaining finite two-point functions of the scalar operators in the corresponding dual gauge theories. The presentation is self-contained and reviews all the relevant background material concerning a gauge-invariant description of the fluctuations around holographic renormalization group backgrounds.

The many papers presented in this special edition should be very useful to beginning researchers and graduate students specializing in high-energy theory and in theoretical condensed matter physics. We would like to thank all contributors for their very hard work and patience in bringing out this volume.

Wolfgang Mück Carlos Núñez Leopoldo A. Pando Zayas

Alfonso V. Ramallo

Radoslav C. Rashkov

Kadayam S. Viswanathan 

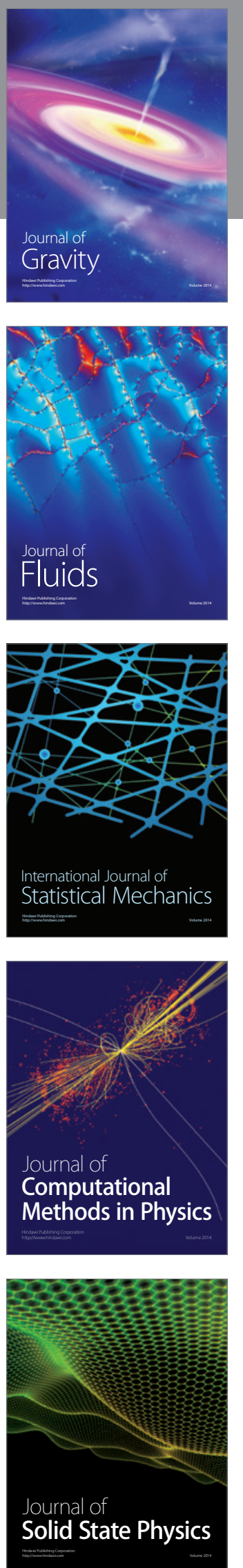

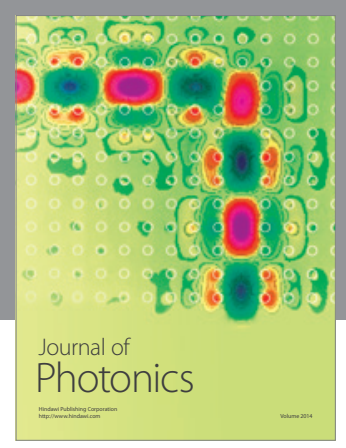

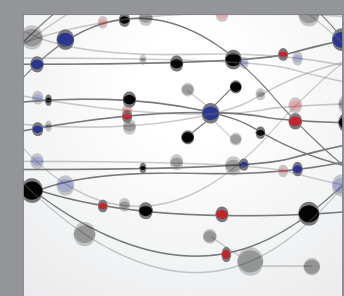

The Scientific World Journal
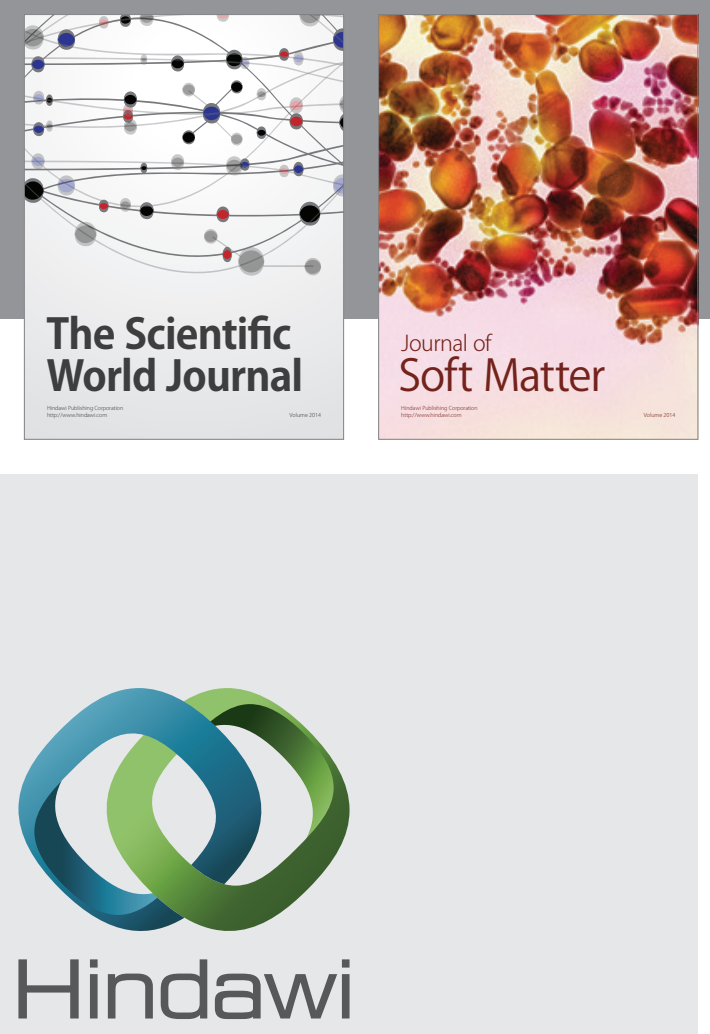

Submit your manuscripts at

http://www.hindawi.com
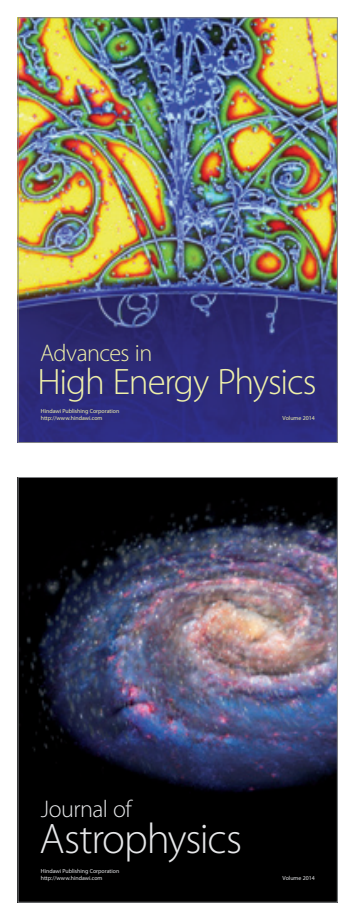
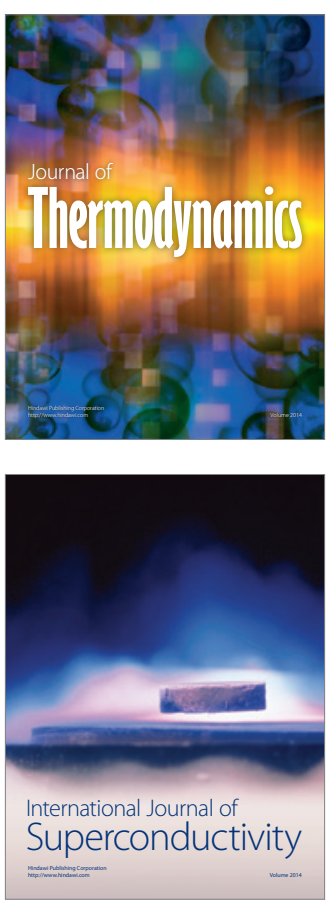
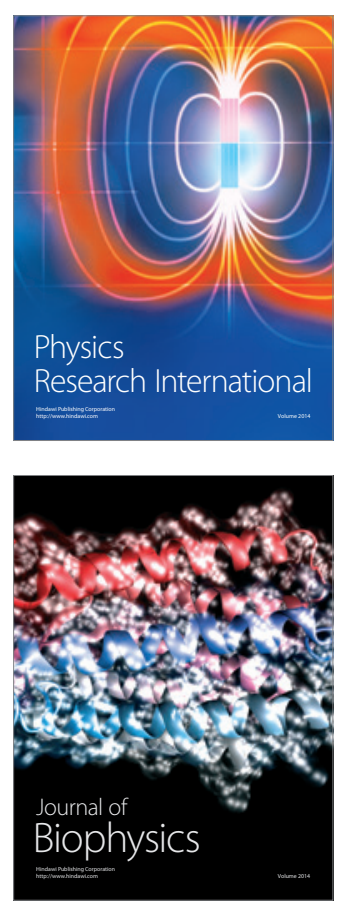
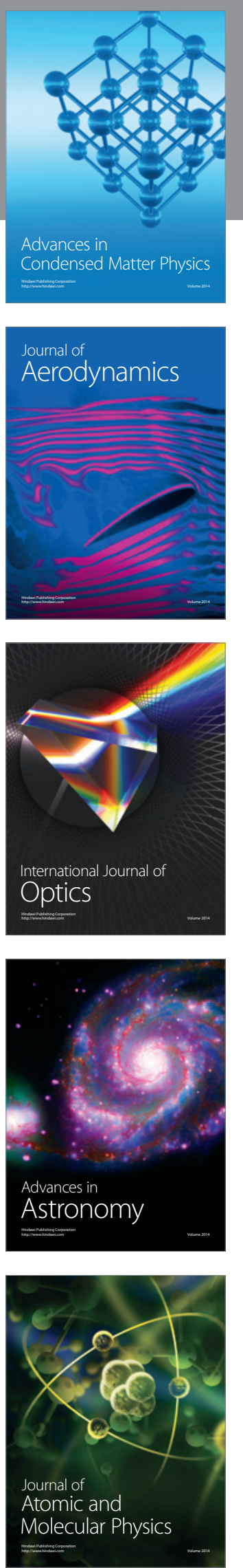